Waugh.A,. Gugjonsson, G.H., Rees-Jones, A., \& Young, S. (2014). A feasibility study of the Reasoning and Rehabilitation Mental Health Programme (R\&R2MHP) in male offenders with intellectual disability. Criminal Behaviour and Mental Health, 24, 222-

\title{
A feasibility study of the Reasoning and Rehabilitation Mental Health Programme (R\&R2MHP) in male offenders with intellectual disability
}

A Prison Reform Trust 2007 briefing paper (Loucks, 2007) showed that intellectual disability (ID) offenders are likely to struggle to complete mainstream offending behaviour programmes due to the cognitive or functional deficits associated with their ID. This has been supported in research showing that mainstream offending behaviour programmes are inappropriate and ineffective for use with ID offenders (Jones, 2007). A review of the psychological interventions currently available to violent offenders with ID within secure hospitals and community settings suggests that there has been an improvement in reducing anger problems in offenders with ID (Taylor and Novaco, 2005; Bond, 2012). Furthermore, Lindsay et al. (2011) found evidence that the Social Problem Solving and Offence Related Thinking Programme was effective in improving the social problem solving styles of people with ID. This finding is particularly relevant to our own work with mentally disordered offenders (MDOs) with ID.

We conducted a pilot study of the revised Reasoning and Rehabilitation (R\&R) Programme developed for delivery to MDOs [R\&R Mental Health Programme (R\&R2MHP); Young and Ross, 2007a] to ascertain whether it was feasible to deliver the programme to inpatient MDOs with ID by assessing programme completion. We hypothesised that the R\&R2MHP would be suitable for ID offenders with completion rates comparable with the $78 \%$ obtained when delivered to inpatient MDOs of normal intelligence (Rees-Jones et al., 2012), which was substantially higher than the 50\% reported for the original R\&R Programme (Cullen et al., 2011) when delivered to a similar population. Hodgins et al. (2011), using the original $R \& R$ in community patients, found that none of the 28 men completed the programme, and the authors recommend that patients should be paid for attending the programme in the community.

In the present study, 25 inpatients were recruited from two medium secure hospital units for male MDOs with ID and received the group intervention. 
The mean Full Scale IQ (FSIQ) was 65.82 (range $=52-70$ ). R\&R2MHP is a 16week, 90-minute group intervention provided once per week. It features a module to address cognitive impairments, specifically attention, memory, impulsiveness and constructive planning and includes a manualised 'coaching' programme where patients meet with a member of staff between group sessions to help them transfer acquired skills to daily life. Some minor adaptations were made to the programme to make it applicable for patients with ID. Treatment completion was set at $80 \%$ group attendance. Participants who attended fewer than 12 sessions were classified as non-completers.

Twenty-three (92\%) of the participants completed the programme. This supports our hypothesis that the programme is feasible for use for offenders with ID. The easily comprehensible content and user friendly presentation of the R\&R2MHP may have a special appeal for ID offenders. The R\&R2MHP was considered appropriate for the ID group in the current study because the participants were in a mental health setting. However, there is a parallel programme available for people with attention deficit and hyperactivity disorder (Young and Ross, 2007b; R\&R2ADHD), which has proved effective in reducing attention deficit and hyperactivity disorder symptoms and co-morbid problems (Emilsson et al., 2011; Young et al., 2012). Future research among ID offenders should compare the strengths and weaknesses of the two programmes with ID offender populations.

\section{ALISON WAUGH \\ PROFESSOR GISLI H. GUDJONSSON DR. ANGHARAD REES-JONES AND DR. SUSAN YOUNG}

\section{References}

Bond N (2012) A literature review of psychological interventions with violent offenders with intellectual difficulties. Forensic Update 105: 29-33.

Cullen AE, Soria C, Clarke A, Dean K, Fahy T (2011) Factors predicting dropout from the reasoning and rehabilitation programme with mentally disordered offenders. Criminal Justice and Behaviour 38: 217-230.

Emilsson B, Gudjonsson G, Sigurdsson JF, Baldursson G, Einarsson E, Olafsdottir H, Young S (2011) Cognitive behaviour therapy in medication treated adults with ADHD and persistent symptoms: a randomised controlled trial. BMC Psychiatry 11: 116.

Hodgins S, Carlin P, Moorhouse R, Legge K, Khalid F (2011) Letter to the editor. Reducing antisocial behaviour among patients with severe mental illness living in the community: a feasibility study of the reasoning and rehabilitation programme. Criminal Behaviour and Mental Health 21: 75-76.

Jones J (2007) Persons with intellectual disabilities in the Criminal Justice System: review of issues. International Journal of Offender Therapy and Comparative Criminology 51: 723-733.

Lindsay WR, Hamilton C, Moulton S, Scott S, Doyle M, McMurran M (2011) Assessment and treatment of social problem solving in offenders with intellectual disability. Psychology, Crime \& Law 17: 181-197. 
Loucks N (2007) The prevalence and associated needs of offenders with learning difficulties and learning disabilities. Prison Reform Trust. http://www.prisonreformtrust.org.uk/Publications/ $\mathrm{vw} / 1 / \mathrm{ItemID} / 85$

Rees-Jones A, Gudjonsson GH, Young S (2012) A multisite controlled study of a cognitive skills programme for mentally disordered offenders. BMC Psychiatry 12: 44.

Taylor JL, Novaco RW (2005) Anger Treatment for People With Developmental Disabilities. Chicester, England: Wiley.

Young SJ, Ross RR (2007a) R\&R2 for Youths and Adults with Mental Health Problems: A Prosocial Competence Training Program. Ottawa: Cognitive Centre of Canada (www. cognitivecentre.ca)

Young SJ, Ross RR (2007b) R\&R2 for ADHD Youths and Adults: A Prosocial Competence Train-ing Program. Ottawa: Cognitive Centre of Canada (www.cognitivecentre.ca)

Young SJ, Hopkins G, Perkins D, Farr C, Doidge A, Gudjonsson G (2012) A controlled trial of a cognitive skills program for personality-disordered offenders. Journal of Attention Disorders, accessed online at http://jad.sagepub.com/content/early/2012/02/03/1087054711430333

Address correspondence to: Dr Susan Young, Centre for Mental Health, Division of Brain Sciences, Imperial College London, Claybrook Centre, Charing Cross Campus, 37 Claybrook Road, London W6 8LN, UK. Email: susan.young1@imperial.ac.uk 